Instrumental Achievements

\title{
Crystal Structures of Q-35 Dihydrate and Monohydrate
}

\author{
Yoshiharu Nawata, Keiko Sato, Masafumi Fukushima and Hiroyuki Nagano \\ Research Laboratories, Chugai Pharmaceutical Co. Ltd., Takada, Toshima, Tokyo 171, Japan
}

Q-35 [(rac)-1-cyclopropyl-6-fluoro-1,4-dihydro-8methoxy-7-(3'-methylaminiopiperidin-1'-yl)-4-oxoquinoline-3-carboxylate)] is a new fluoroquinolone ${ }^{1}$ which exhibits a broad spectrum of antimicrobial activity. ${ }^{2}$ The crystal structure determinations of the title compounds were undertaken to elucidate the threedimensional structure around the water molecules in both crystals.

Dihydrate (I) (colorless thin plates) was grown from an aqueous solution, and monohydrate (II) (colorless needles) was grown from a methanol $/ \mathrm{H}_{2} \mathrm{O}(\mathrm{v} / \mathrm{v}=10: 1)$ solution. Experimental conditions: crystal size $0.40 \mathrm{X}$ $0.23 \times 0.03 \mathrm{~mm}$ for (I) and $0.18 \times 0.15 \times 0.05 \mathrm{~mm}$ for (II), $\mathrm{Cu} \mathrm{K} \mathrm{K}_{\alpha}$ radiation (graphite-monochromated), $\theta-2 \theta$ scan with variable scan speed $1.27-2.75 \% \min (\theta)$ for (I) and $1.10-4.12 \% \min (\theta)$ for (II), scan width $(0.60+0.14 \tan \theta)^{\circ}$ for (I) and $(0.70+0.14 \tan \theta)^{\circ}$ for (II), range of indices

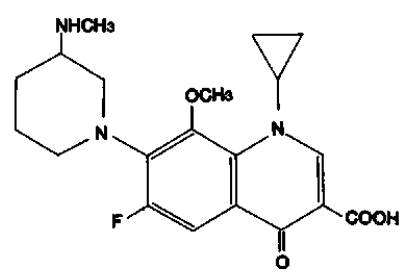

Fig. 1 Chemical structure of Q-35.
$-28 \leqq h \leqq 28,0 \leqq k \leqq 16,0 \leqq l \leqq 8\left(0<\theta<75^{\circ}\right)$ for (I) and $-14 \leqq h \leqq 14,0 \leqq k \leqq 19,0 \leqq l \leqq 12\left(0<\theta<75^{\circ}\right)$ for (II). Lattice constants were determined based on twenty-five $2 \theta$ values $\left[18<\theta<40^{\circ}\right.$ for (I) and $14<\theta<41^{\circ}$ for (II)]. No significant intensity changes were observed during data collection. A total of 4423 unique reflections were collected for (I) and 4194 for (II).

Both structures were solved by direct methods with MULTAN $11 / 82$, and refined by a full-matrix least squares method. All hydrogen atoms for (I) were found on D-Fourier maps, and those for (II) were calculated stereochemically, except for those of iminio group and water. Non-H atoms were refined with anisotropic thermal parameters and $\mathrm{H}$ atoms with the isotropic ones (fixed at $B=5.0 \AA^{2}$ ). The quantity $\sum w\left(\left|F_{\mathrm{o}}\right|-\left|F_{\mathrm{c}}\right|\right)^{2}$ was minimized; $w=1.0$ for $\left|F_{0}\right|<F_{\text {thres }}, w=\left(F_{\text {thres }} / F_{\mathrm{o}}\right)^{2}$ for $\left|F_{\mathrm{o}}\right| \geqq F_{\text {thres }}\left[F_{\text {thres }}=149.6\right.$ for (I), and 197.8 for (II)]. The final refinement on (I) yielded the values: $R=0.044$, $w R=0.044, S=2.35$ for 384 variables, secondaryextinction factor $(g) 9(1) \times 10^{-7}\left[\left|F_{\mathrm{o}}\right|=\left|F_{\mathrm{c}}\right| /\left(1+g I_{\mathrm{c}}\right)\right],(\Delta /$ $\sigma) \leqq 0.07$. Corresponding values for (II): $R=0.067$, $w R=0.062, S=3.64$ for 263 variables. The highest peak in the final D-Fourier map was $+0.35 \mathrm{e}^{-3}$ for (I), and $+0.43 \mathrm{e}^{-3}$ for (II).

The crystal and experimental data are given in Table 1 and the final atomic parameters in Table 2. The molecular structures are shown in Figs. 2(a) and (b),

Table 1 Crystal and experimental data of Q-35 Hydrates

\begin{tabular}{|c|c|c|c|}
\hline & Dihydrate & & Monohydrate \\
\hline Formula & $\mathrm{C}_{20} \mathrm{H}_{24} \mathrm{FN}_{3} \mathrm{O}_{4} \cdot 2 \mathrm{H}_{2} \mathrm{O}$ & & $\mathrm{C}_{20} \mathrm{H}_{24} \mathrm{FN}_{3} \mathrm{O}_{4} \cdot \mathrm{H}_{2} \mathrm{O}$ \\
\hline Formula weight & 425.46 & & 407.45 \\
\hline Crystal system & & monoclinic & \\
\hline $\begin{array}{l}\text { Space group } \\
a(\AA)\end{array}$ & $\begin{array}{l}P 2_{1} / a, Z=4 \\
22.984(1)\end{array}$ & & $\begin{array}{l}P 2_{1} / c, Z=4 \\
11.448(1)\end{array}$ \\
\hline$b$ & $12.978(4)$ & & $15.902(1)$ \\
\hline$c$ & $6.867(1)$ & & $10.888(1)$ \\
\hline$\beta\left(^{\circ}\right)$ & $91.19(1)$ & & $90.88(1)$ \\
\hline$V\left(\AA^{3}\right)$ & 2048.0 & & 1981.9 \\
\hline$D_{\mathrm{x}}\left(\mathrm{g} \mathrm{cm}^{-3}\right)$ & 1.380 & & 1.365 \\
\hline$R$ & 0.044 & & 0.065 \\
\hline No. of reflections used & 2642 & & 2616 \\
\hline Measurement & & Enraf-Nonius CAD4 & \\
\hline Program system & & Enraf-Nonius SDP & \\
\hline Structure determination & & MULTAN 11/82 & \\
\hline Refinement & & full matrix & \\
\hline
\end{tabular}


Table 2 Final atomic coordinates and equivalent isotropic temperature fractors $B_{\text {eq }}$

\begin{tabular}{|c|c|c|c|c|c|c|c|c|c|}
\hline Atom & $x$ & $y$ & $z$ & $B_{\mathrm{eq}} / \AA^{2}$ & Atom & $x$ & $y$ & $z$ & $B_{\text {eq }} / \AA^{2}$ \\
\hline \multicolumn{5}{|c|}{ (a) Dihydrate } & \multicolumn{5}{|c|}{ (b) Monohydrate } \\
\hline$n(1)$ & $0.45530(9)$ & $0.3092(2)$ & $1.4759(3)$ & $3.81(4)$ & $n(1)$ & $0.8870(3)$ & $0.97 .34(2)$ & $0.4779(4)$ & $4.59(9)$ \\
\hline$C(2)$ & $0.4511(\mathrm{~J})$ & $0.2 .041(2)$ & $1.4466(4)$ & $2.83(6)$ & $r(2)$ & $0.8589(5)$ & $0.8475(4)$ & $0.4662 .(5)$ & $3.9(3)$ \\
\hline $0(3)$ & $0.48154(9)$ & $0.2 .7 .12 .2(2)$ & $1.5314(3)$ & $3.73(5)$ & $n(3)$ & $0.897 .8(4)$ & $0.8023(3)$ & $0.382 .8(4)$ & $6.3(1)$ \\
\hline$C(4)$ & $0.4069(3)$ & $0.2 .412(2)$. & $1.2952 .(4)$ & $2.47(5)$ & $C(4)$ & $0.7749(4)$ & $0.8135(4)$ & $0.5587(5)$ & $3.2(1)$ \\
\hline$C(5)$ & $0.3887(\mathrm{~J})$ & $0.1818(2)$ & $1.1300(4)$ & $2.35(5)$ & $C(5)$ & $0.77 .39(4)$ & $0.8574(4)$ & $0.6560(5)$ & $3.6(1)$ \\
\hline$n(6)$ & $0.40593(9)$ & $0.0938(2)$ & $1.0904(3)$ & $3.10(4)$ & $0(6)$ & $0.7439(4)$ & $0.932 .7 .(3)$ & $0.6833(4)$ & $5.8(1)$ \\
\hline$c(7)$ & $0.3463(1)$ & $0.2 .342(2)$. & $1.0003(4)$ & $2.21(5)$ & $C(7)$ & $0.6357(4)$ & $0.8113(4)$ & $0.72 .56(5)$ & $3.0(3)$ \\
\hline$C(8)$ & $0.3257(1)$ & $0.1780(2)$ & $0.8391(4)$ & $2.79(6)$ & $r(8)$ & $0.5684(4)$ & $0.8577 .(4)$ & $0.8077(5)$ & $3.4(3)$ \\
\hline$c(9)$ & $0.2831(1)$ & $0.2 .187(2)$. & $0.7237(4)$ & $2.77(6)$ & $r(9)$ & $0.4815(4)$ & $0.8191(4)$ & $0.8690(5)$ & $3.7(1)$ \\
\hline$F(10)$ & $0.26092(8)$ & $0.1609(2)$ & $0.5733(3)$ & $4.16(4)$ & $F(10)$ & 0.4 .77 .2 .31 & $0.8660(2)$ & $0.947 .1(3)$ & $4.89(8)$ \\
\hline$c(11)$ & $0.2572 .(1)$ & $0.3147(2)$ & $0.7568(4)$ & $2.42 .(5)$ & (1) & $0.4578(4)$ & $0.732 .2 .(4)$ & $0.8581(5)$ & $3.1(1)$ \\
\hline$N(12)$ & $0.2084(1)$ & $0.3432 .(2)$ & $0.647 .5(3)$ & $2.97(5)$ & $N(12)$ & $0.3636(3)$ & $0.6998(3)$ & $0.92 .09(4)$ & $3.30(9)$ \\
\hline$r(13)$ & $0.2 .136(1)$ & $0.3580(3)$ & $0.4335(4)$ & $3.67(7)$ & $(1,3)$ & $0.3573(6)$ & $0.7051(5)$ & $3.0529(5)$ & $5.9(2)$ \\
\hline$C(14)$ & $0.1587(2)$ & $0.3260(3)$ & $0.32 .60(4)$ & $4.29(8)$ & $C(14)$ & $0.2 .42 .4(6)$ & $0.7048(5)$ & $1.1010(6)$ & $6.5(2)$. \\
\hline$C(15)$ & $0.1060(1)$ & $0.3796(3)$ & $0.4087(4)$ & $3.44(7)$ & $c(15)$ & $0.3643(5)$ & $0.636 .1(4)$ & $3.0501(5)$ & $4.3(1)$ \\
\hline$r(16)$ & $0.3037(3)$ & $0.3605(2)$ & $0.62 .70(4)$ & $2.77(6)$ & $c(16)$ & $0.372 .3(5)$ & $0.6353(4)$ & $0.9100(5)$ & $4.9(3)$ \\
\hline$N(17)$ & $0.05257(9)$ & $0.4] 46(2)$ & $0.7303(3)$ & $2.4](4)$ & $N(17)$ & $0.0988(3)$ & $0.5649(3)$ & $0,8618(4)$ & $3.39(9)$ \\
\hline$C(18)$ & $0.3593(3)$ & $0.3962(3)$ & $0.72 .76(4)$ & $3.4 .3(7)$ & $\Gamma(18)$ & $0.2 .9 .11(5)$ & $0.63 .17(5)$ & $0.8654(5)$ & $5.0(1)$ \\
\hline$C(19)$ & $0.2808(1)$ & $0.3740(2)$ & $0.9093(4)$ & $2.2 .4(5)$ & $r(19)$ & $0.52 .97 .(4)$ & $0.6857(4)$ & $0.7835(5)$ & $3.3(3)$ \\
\hline$n(20)$ & $0.25803(8)$ & $0.4700(2)$ & $0.944 .3(3)$ & $2.86(4)$ & $n(20)$ & $0.512 .6(3)$ & $0.6003(2)$ & $0.7746(3)$ & $3.72(8)$ \\
\hline$C(2.1)$ & $0.3255(1)$ & $0.3343(2)$. & $1.0346(4)$ & $2.33(5)$ & $r(2.1)$ & $0.6166(4)$ & $0.72 .45(4)$ & $0.7106(5)$ & $3.0(1)$ \\
\hline$N(2.2)$ & $0.34952 .(9)$ & $0.3890(2)$ & $1.1934(3)$ & $2.2 .6(4)$ & $N(2.7)$. & $0.6821(4)$ & $0.682 .3(3)$ & $0.62 .28(4)$ & $3.30(9)$ \\
\hline$c(2.3)$ & $0.3372(1)$ & $0.4974(2)$ & $1.2367(5)$ & $2.81(6)$ & $c(2.3)$ & $0.6754(5)$ & $0.5899(4)$ & $0.602 .3(5)$ & $3.9(1)$ \\
\hline$C(2.4)$ & $0.3662 .(2)$ & $0.587 .9(3)$ & $1.1326(5)$ & $4.03(7)$ & $C(2.4)$ & $0.7468(6)$ & $0.5329(4)$ & $0.6773(6)$ & $5.1(2)$ \\
\hline$C(7.5)$ & $0.3825(2)$ & $0.5611(3)$ & $1.3398(5)$ & $4.43(8)$ & $C(2.5)$ & $0.7738(6)$ & $0.5466(4)$ & $0.5447(6)$ & $5.3(2)$ \\
\hline$r(26)$ & $0.3873(1)$ & $0.3394(2)$ & $1.3146(4)$ & $2.63(5)$ & $C(2.6)$ & $0.752 .4(4)$ & $0.72 .77 .(4)$ & $0.5492 .(5)$ & $3.5(1)$ \\
\hline$r(2.7)$ & $0.0373(1)$ & $0.3762 .(3)$ & $0.9059(5)$ & $3.46(7)$ & $r(2.7)$ & $0.0782(5)$ & $0.5682 .(5)$ & $0.72 .69(6)$ & $5.3(2)$ \\
\hline$C(28)$ & $0.2713(3)$. & $0.54 .50(3)$ & $0.7969(5)$ & $4.28(8)$ & $c(2.8)$ & $0.5539(6)$ & $0.5554(4)$ & $0.8815(6)$ & $5.2(2)$ \\
\hline ถW1 & $0.52 .97 .(\mathrm{J})$ & $0.3946(3)$ & $3.2220(5)$ & $7.33(8)$ & nN & $0.997 .5(7)$ & $0.6492 .(5)$ & $0.3361(9)$ & $16.0(3)$ \\
\hline กพ2 & $0.5377(2)$ & $0.6072 .(2)$ & $1.3357(4)$ & $7.14(8)$ & & & & & \\
\hline
\end{tabular}
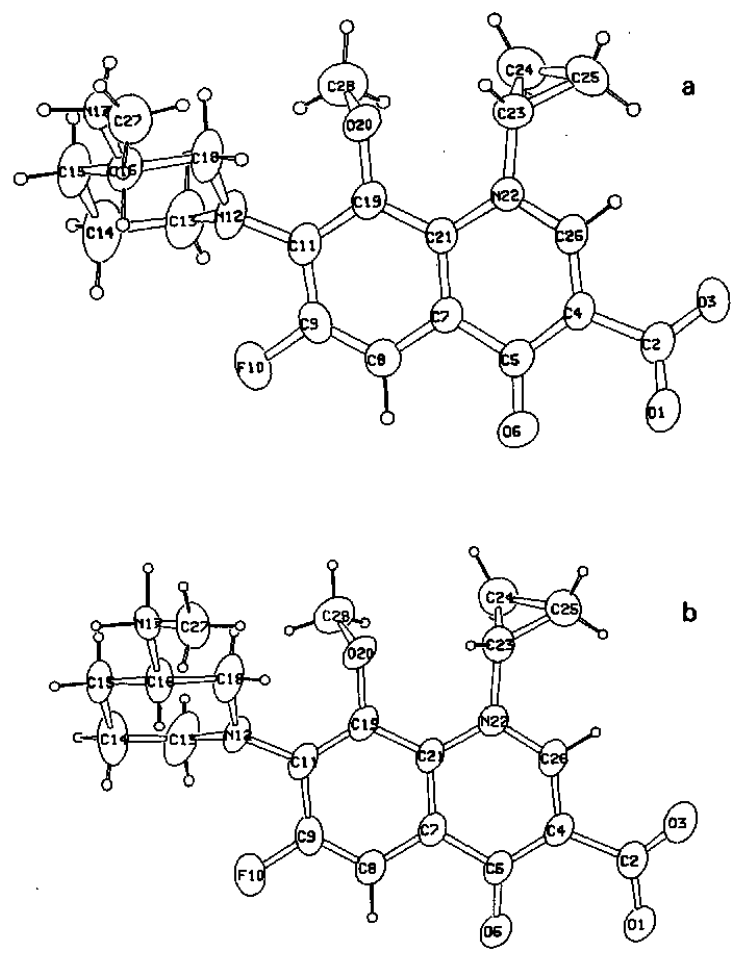

together with the atomic numbering scheme. The molecular conformations of Q-35 in both crystals are approximately identical. Intermolecular interactions, including electrostatic and hydrogen bonds, are observed between the iminio $(\mathrm{N}(17))$ and the carboxylate $(\mathrm{O}(1)$, $\mathrm{O}(3)$ ) groups in both crystals. Each ionic center is linked to the two opposite ionic centers of the different molecules to form a three-dimensional network. In (I) four water molecules, which surround a center of symmetry, are linked to each other by hydrogen bonds to form an eight-membered ring. These eight-membered rings are packed in the tunnels that are running through the crystal along the $c$-axis and piled up to form octagonal columns held by carboxylate $O(3)$. On the contrary, each water molecule in (II) is near enough to the carboxylate $\mathrm{O}(3)$ to form a hydrogen bond: OW... $\mathrm{O}=2.740(9) \AA, \mathrm{HW} \cdots \mathrm{O}=1.73 \AA$, OW-HW $\cdots \mathrm{O}=154^{\circ}$.

\section{References}

1. Y. Nagano, T. Yokota and Y. Kato, Japanese Patent 395177 (1991).

2. T. Ito, M. Otsuki and T. Nishino, Antimicrob. Agents Chemother., 36, 1708 (1992).

Fig. 2 Molecular structure of Q-35 (a) in (I) and (b) in (II). 